\title{
Criminologie
}

\section{Modèle interactif du risque suicidaire chez les jeunes contrevenants}

\section{Catherine Laurier et François Chagnon}

Volume 44, numéro 2, automne 2011

URI : https://id.erudit.org/iderudit/1005799ar

DOI : https://doi.org/10.7202/1005799ar

Aller au sommaire du numéro

\section{Éditeur(s)}

Les Presses de l’Université de Montréal

ISSN

0316-0041 (imprimé)

1492-1367 (numérique)

Découvrir la revue

Citer cet article

Laurier, C. \& Chagnon, F. (2011). Modèle interactif du risque suicidaire chez les jeunes contrevenants. Criminologie, 44(2), 251-278.

https://doi.org/10.7202/1005799ar
Résumé de l'article

Bien que l'on observe une diminution marquée de la mortalité par suicide chez les jeunes au cours des dernières années, le suicide représente la deuxième cause de mortalité chez les 15-19 ans et la première chez les 20-34 ans. Les délinquants juvéniles constituent un groupe à risque accru de comportements suicidaires, mais les facteurs de risque chez cette population sont encore peu étudiés. Cet article présente les principaux facteurs de risque et de protection associés au risque suicidaire chez les jeunes contrevenants en les intégrant dans un modèle interactif. Parmi les facteurs pris en compte dans le modèle se trouvent les facteurs liés aux événements de vie, les facteurs individuels (psychopathologie, consommation de substances psychotropes et/ou d'alcool, caractéristiques individuelles et de personnalité), les facteurs sociaux (famille, réseau social) et les facteurs résidentiels (milieu de vie et voisinage, école, milieu résidentiel pour jeunes contrevenants). Afin d'appuyer le modèle interactif présenté, le cas d'un adolescent jeune contrevenant, Olivier, est exposé. 


\title{
Modèle interactif du risque suicidaire chez les jeunes contrevenants
}

\author{
Catherine Laurier \\ Chercheure \\ Centre jeunesse de Montréal - Institut universitaire \\ Professeure associée \\ École de criminologie, Université de Montréal \\ Centre de recherche sur le suicide et l'euthanasie (Crise) \\ catherine.laurier@cjm-iu.qc.ca \\ François Chagnon \\ Professeur \\ Département de psychologie, Université du Québec à Montréal (UQAM) \\ Centre de recherche sur le suicide et l'euthanasie (Crise) \\ Chaire d'étude CJM-IU sur l'application des connaissances dans le domaine des jeunes et \\ des familles en difficulté \\ chagnon.francois@uqam.ca
}

RÉSUMÉ • Bien que l'on observe une diminution marquée de la mortalité par suicide chez les jeunes au cours des dernières années, le suicide représente la deuxième cause de mortalité chez les 15-19 ans et la première chez les 20-34 ans. Les délinquants juvéniles constituent un groupe à risque accru de comportements suicidaires, mais les facteurs de risque chez cette population sont encore peu étudiés. Cet article présente les principaux facteurs de risque et de protection associés au risque suicidaire chez les jeunes contrevenants en les intégrant dans un modèle interactif. Parmi les facteurs pris en compte dans le modèle se trouvent les facteurs liés aux événements de vie, les facteurs individuels (psychopathologie, consommation de substances psychotropes et/ou d'alcool, caractéristiques individuelles et de personnalité), les facteurs sociaux (famille, réseau social) et les facteurs résidentiels (milieu de vie et voisinage, école, milieu résidentiel pour jeunes contrevenants). Afin d'appuyer le modèle interactif présenté, le cas d'un adolescent jeune contrevenant, Olivier, est exposé.

MOTS-CLÉs - Suicide, adolescents, tentative de suicide, idéations suicidaires, jeunes contrevenants, modèle conceptuel, facteurs de risque.

\section{Introduction}

Au Québec, bien qu'on observe une diminution marquée de la mortalité par suicide chez les jeunes au cours des dernières années, le suicide 
demeure la deuxième cause de mortalité chez les adolescents âgés de 15 à 19 ans et la première cause chez les jeunes adultes de 20 à 34 ans (St-Laurent et Gagné, 2008). Pour l'année 2006, les taux de mortalité par suicide étaient de 7,4/100 000 pour les 15-19 ans et de 14,9/100 000 pour les 20-34 ans (St-Laurent et Gagné, 2008). Plusieurs études, pour la plupart réalisées aux États-Unis (Battle et al., 1993 ; Brent et al., 1993; Brent et al., 1994; Brent et al., 1996; Renaud et al., 1999; Rohde, Mace et al. 1997; Rohde, Seeley et al., 1997; Langhinrichsen-Rohling et al., 2004; Hayes, 2005; Brent et Bridge, 2007; Abram et al., 2008) et en Nouvelle-Zélande (Beautrais, 2000, 2001, 2002, 2003a; Fergusson et al., 2005) ont examiné les facteurs associés aux comportements suicidaires chez les adolescents.

Tout comme c'est le cas pour les adultes, ces études montrent que la psychopathologie est fortement associée aux idéations suicidaires, aux tentatives de suicide et aux suicides complétés. En ce domaine, il est à noter qu'au-delà des troubles intériorisés (dépression et anxiété), les troubles extériorisés (notamment les troubles du comportement, l'agressivité et la délinquance) occupent une place importante dans la compréhension des comportements suicidaires. Plus encore, les adolescents délinquants présentant des troubles de la conduite représentent une population particulièrement à risque de suicide. En effet, aux ÉtatsUnis, le risque de suicide complété au sein de la population délinquante juvénile $(57 / 100000)$ serait 4,6 fois supérieur à ce qui est retrouvé dans la population générale (Memory, 1989).

Les études effectuées au Québec concernant le suicide et les comportements suicidaires chez les adolescents délinquants sont rares ${ }^{1}$. Elles confirment toutefois les données retrouvées aux États-Unis selon lesquelles les délinquants juvéniles présentent un risque accru de comportements suicidaires.

L'étude de Farand et al. réalisée au Québec en 2004 a mis de l'avant que les adolescents pris en charge par les centres jeunesse au Québec

1. Il est cependant essentiel de garder à l'esprit que la notion même de délinquance est difficile à définir et à cerner. Dans certains cas, les études considèrent comme «délinquants» uniquement les jeunes qui ont commis un délit au sens de la loi alors que dans d'autres cas, il s'agit d'une délinquance auto-révélée par questionnaires. Selon les États et les provinces, la définition change aussi. De même, la délinquance n'est pas un trouble faisant partie du DSM-IV où l'on fait plutôt référence à un trouble des conduites. Dans ce texte, par rapport aux lois en vigueur au Québec, nous considérerons comme «délinquants» les adolescents pris actuellement en charge ou l'ayant été dans le passé selon la Loi sur le système de justice pénale pour adolescents. 
risquent quatre fois plus de se suicider que les autres. De ces jeunes, ceux qui sont pris en charge par la Loi des jeunes contrevenants (LJC - loi en vigueur au moment de la recherche) ou qui sont hébergés en raison de troubles du comportement (article $38 \mathrm{~h}$ de la Loi sur la protection de la jeunesse - LPJ) sont encore plus vulnérables, car ils présentent un risque de se suicider 8,3 fois supérieur à celui des adolescents du Québec qui ne sont pas pris en charge selon ces lois (Farand et al., 2004). Ces résultats doivent cependant être interprétés avec prudence puisqu'ils ne concernent que les jeunes ayant commis un suicide au cours des années 1995-1996 $(\mathrm{N}=177)$ et seulement 57 d'entre eux avaient un dossier aux centres jeunesse du Québec. Néanmoins, ils suggèrent que les comportements délinquants pourraient constituer un facteur associé à un risque suicidaire accru, possiblement en raison de la personnalité vulnérable de ces jeunes combinée à leurs vicissitudes biographiques, les ayant d'ailleurs conduits à être pris en charge par les centres jeunesse.

Outre le suicide, les jeunes hébergés en vertu de la Loi sur la protection de la jeunesse (LPJ) ou des jeunes contrevenants (Loi sur le système de justice pénale pour adolescents ou Loi sur les jeunes contrevenants, selon l'année de l'étude) présentent un risque important de tentative de suicide et d'idéation suicidaire. L'étude de Chagnon (2000) réalisée auprès de 210 adolescents admis au Centre jeunesse de Montréal - Institut universitaire révèle que $35 \%$ rapportent au moins une tentative de suicide au cours de leur vie. Une autre recherche effectuée au Québec parmi 435 jeunes hébergés dans les centres jeunesse arrive à des résultats similaires: $68 \%$ étant dépistés à risque de suicide (Pronovost et Leclerc, 2002). Aux États-Unis, selon les études concernées, de $16 \%$ à $61 \%$ des adolescents incarcérés révèlent avoir fait une tentative de suicide au cours de la dernière année (Alessi et al. 1984; Dembo et al., 1990; Kempton et Forehand, 1992; Morris et al., 1995; Esposito et Clum, 2002; Howard et al., 2003); de 13,5\% à 52\% auraient des comportements ou des idéations suicidaires actuels (Davis et al., 1991; Morris et al., 1995; Esposito et Clum, 2002). Pronovost et Leclerc (2002) ont étudié les principaux éléments déclencheurs des événements suicidaires des jeunes hébergés en centres jeunesse. Ainsi, $46 \%$ des jeunes ( $42,2 \%$ des filles et $49,6 \%$ des garçons) ayant rapporté un ou plusieurs événements suicidaires mentionnent que ceux-ci ont été déclenchés par une situation liée au fait d'être placés en centres jeunesse. Ces résultats suggèrent que la prise en charge de l'adolescent délin- 
quant, bien qu'elle soit nécessaire, peut contribuer à amplifier le risque suicidaire.

Malgré le risque élevé de suicide que présentent les jeunes délinquants, les facteurs de risque de suicide chez ces jeunes demeurent peu connus, tout comme ce qui différencie les délinquants suicidaires des non suicidaires. Cette méconnaissance représente un obstacle important à la prévention du suicide chez ces jeunes.

Cet article a pour objectif de présenter les principaux facteurs de risque et de protection associés au risque suicidaire chez les jeunes contrevenants en les intégrant dans un modèle explicatif. On y présente d'abord une revue selon deux principaux groupes de facteurs de risque: 1) les facteurs liés aux événements de vie; et 2) les facteurs associés et de protection. En deuxième partie, un modèle explicatif du suicide chez les délinquants est exposé. En appui à l'application du modèle, une vignette clinique est présentée.

\section{De multiples facteurs de risque suicidaire chez les jeunes contrevenants}

Les facteurs de risque du suicide chez les jeunes contrevenants sont multiples et liés entre eux. Nous aborderons en premier les facteurs liés aux événements de vie (lointains et récents). Dans un deuxième temps, nous aborderons les facteurs individuels, soit la psychopathologie, la consommation de drogues et d'alcool, les caractéristiques individuelles et de personnalité. Troisièmement, il sera question des facteurs sociaux, comprenant l'influence de la famille et du réseau social sur la délinquance et les comportements suicidaires. Finalement, les facteurs environnementaux seront abordés; il y sera question du voisinage, de l'école, ainsi que de l'influence que peut avoir le milieu résidentiel pour jeunes contrevenants sur la compréhension de la problématique du suicide.

\section{Facteurs liés aux événements de vie}

Événements du passé lointain

Les événements de vie difficiles et stressants dans le passé des adolescents peuvent contribuer à la fois à l'instauration de la délinquance et des comportements suicidaires. Ainsi en est-il de la prise en charge par les centres jeunesse (Farand et al., 2004) ou les «foster care» (Ryan et 
Testa, 2005; Alltucker et al., 2006) et les abus sexuels et/ou physiques dans l'enfance (Alessi et al., 1984; Battle et al., 1993; Zingraff et al., 1993 ; Rohde, Mace et al., 1997; Rohde, Seeley et al., 1997; Beautrais, 2000; American Academy of Child and Adolescent Psychiatry, 2001; Chagnon et al., 2001; Esposito et Clum, 2002; Flouri et Buchanan, 2002; Beautrais, 2003a; Howard et al., 2003 ; Penn et al., 2003; Flouri, 2005; Hayes, 2005 ; Ryan et Testa, 2005 ; Daigle et Côté, 2006; Brent et Bridge, 2007). Nous savons en effet que le fait d'être séparé de son milieu familial indique la présence de problèmes qui peuvent conduire à la compromission du développement de l'enfant, ce qui peut constituer en amont un risque d'apparition de troubles d'adaptation (Alltucker et al., 2006; Christoffersen et al., 2007). De même, avoir été séparé de son milieu d'origine familial augmente le risque d'un problème de délinquance. Outre ces sources de stress au quotidien, une étude récente établit un lien entre les expériences traumatiques et les idéations suicidaires chez les adolescents détenus (Chapman et Ford, 2008).

Davantage que l'influence relative d'un événement par rapport à un autre, c'est l'accumulation des situations difficiles dans l'enfance qui augmenterait le risque de délinquance (DeMatteo et Marczyk, 2005; Lay et al., 2005). En effet, ces situations difficiles sont des sources de stress pour l'enfant et peuvent s'accumuler et ainsi le rendre plus vulnérable aux autres facteurs de risque qui peuvent se présenter par la suite. Suivant cette même logique causale, le risque de tentative de suicide augmenterait d'autant plus que le nombre d'expériences négatives dans l'enfance est élevé (Dube et al., 2001). Ainsi, le cumul chez un enfant de situations difficiles générant un stress élevé augmenterait le risque de délinquance et les conduites suicidaires.

\section{Événements du passé récent}

Outre les événements de vie difficiles dans le passé éloigné, les événements stressants vécus récemment amplifient le risque de suicide et peuvent déclencher le suicide ou la tentative de suicide (Rohde, Mace et al., 1997; American Academy of Child and Adolescent Psychiatry, 2001 ; Beautrais, 2000, 2003b; Portzky et al., 2005). Les événements récents les plus souvent associés au risque de suicide chez les adolescents sont la perte d'une relation (Woolf et Fink, 1985; Brent et al., 1994; American Academy of Child and Adolescent Psychiatry, 2001; Flouri et Buchanan, 2002; Beautrais, 2003a; Portzky et al., 2005), des 
sanctions disciplinaires ou judiciaires (Woolf et Fink, 1985; Wilson et al., 1995; Beautrais, 2000; American Academy of Child and Adolescent Psychiatry, 2001; Beautrais, 2001; Chagnon et al., 2001; Flouri et Buchanan, 2002; Beautrais, 2003a; Otzenberger, 2003), être sans emploi (Woolf et Fink, 1985; Chagnon et al., 2001; Beautrais, 2003a), ou avoir vécu le suicide d'un membre de la famille ou d'un proche (Flouri et Buchanan, 2002; Pena et Caine, 2006). Les conflits familiaux (Brent et al., 1994; Wilson et al., 1995; Portzky et al., 2005) figurent aussi parmi les facteurs déclencheurs de l'acte suicidaire chez les adolescents, même s'ils peuvent être sans graves conséquences (Beautrais, 2001). Ces conflits sont fréquents dans les familles dont sont issus les jeunes délinquants (Patterson et al., 1989).

Dans le cas des adolescents pris en charge par le système judiciaire, l'ordonnance de placement dans un foyer d'accueil ou centre de réadaptation indique habituellement la réponse donnée à une période de crise (Chagnon et al., 2001; Gallagher et Dobrin, 2006). L'hébergement en centre jeunesse ajouterait un stress supplémentaire à la situation déjà difficile vécue par l'adolescent (Rohde, Seeley et al., 1997; Gallagher et Dobrin, 2006; Wasserman et McReynolds, 2006; Chagnon et al., 2007).

\section{Facteurs individuels}

Psychopathologie

Environ $90 \%$ des personnes décédées par suicide présentent au moins un trouble mental au moment de leur décès (Rohde, Mace et al., 1997; Beautrais, 2000; American Academy of Child and Adolescent Psychiatry, 2001; Beautrais, 2003a). Tant les troubles intériorisés qu'externalisés représentent des facteurs de risque de comportements suicidaires chez les adolescents de la population générale (Sourander et al., 2001; Portzky et al., 2005) et chez ceux qui sont incarcérés (Chowanec et al., 1991) ou hébergés en institution (Hukkanen et al., 2003).

Environ le tiers des adolescents en centre d'évaluation judiciaire présenteraient au moins un trouble psychiatrique (McReynolds et al., 2008; Nolen et al., 2008), ce qui favorise d'autant leur risque suicidaire. La délinquance et la prise en charge qui en résulte peuvent donner lieu à certains troubles (par exemple l'anxiété et la dépression) et à l'aggravation de ceux existant déjà (Goldstein et al., 2005).

Les troubles et symptômes dépressifs sont reconnus comme les troubles psychiatriques les plus souvent associés aux comportements suicidaires 
chez les adolescents (Apter et al., 1988; Fombonne, 1998; Beautrais, 2000; American Academy of Child and Adolescent Psychiatry, 2001; Esposito et Clum, 2002; Flouri et Buchanan, 2002; Beautrais, 2003a, 2003b; Fergusson et al., 2003; Renaud et al., 2005; Connor et Rueter, 2006; Thompson et al., 2007). Pour certains auteurs (Fombonne et al., 2001), l'épisode dépressif majeur constitue un facteur de risque de comportements suicidaires encore plus important quand il est combiné à un trouble des conduites. La dépression est le trouble psychiatrique le plus fréquent chez les jeunes délinquants (Goldstein et al., 2005) (en excluant le trouble des conduites, et elle affecterait environ $80 \%$ des jeunes incarcérés [Domalanta et al., 2003]). Plus spécifiquement, chez des adolescents qui présentent des troubles du comportement ou des conduites, la concomitance des symptômes dépressifs augmente le risque de comportements suicidaires (Davis et al., 1991; Kempton et Forehand, 1992; Battle et al., 1993; Harris et Lennings, 1993 ; Rohde, Mace et al., 1997; Penn et al., 2003; Sanislow et al., 2003; Langhinrichsen-Rohling et al., 2004; Hayes, 2005; Roberts et Bender, 2006; Wasserman et McReynolds, 2006; Renaud et al., 2008). L'étude de Langhinrichsen-Rohling et al. (2004) illustre bien comment dépression, délinquance et comportements suicidaires peuvent être associés et montre que les jeunes ayant eu des démêlés avec le système de justice - à la suite de comportements délinquants - sont plus à risque de présenter des symptômes à la fois dépressifs et suicidaires.

Présenter un trouble anxieux augmente le risque suicidaire chez les adolescents de la population en général (Brent et al., 1993; Esposito et Clum, 2002; Beautrais, 2003a; Vermeiren et al., 2003; Connor et Rueter, 2006) et chez les adolescents délinquants ou hébergés en centres de réadaptation (Rohde, Seeley et al., 1997; Hukkanen et al., 2003 ; Penn et al., 2003).

En somme, l'ensemble de ces données montre que les troubles dépressifs, tels que la dépression majeure et la dysthymie, de même que les symptômes dépressifs tels que la résignation acquise et le désespoir, placent les adolescents délinquants plus à risque de présenter des comportements suicidaires, allant de l'idéation au suicide complété. Il en est de même pour les troubles du comportement et les troubles anxieux.

\section{Consommation de substances psychotropes}

L'abus de substances psychotropes et/ou d'alcool est associé aux comportements suicidaires chez les adolescents de la population générale et chez 
ceux qui présentent des troubles des conduites (Kotila et Lönnqvist, 1988; Flouri et Buchanan, 2002; Perkins et Hartless, 2002; Putnins, 2005).

La consommation de substances psychotropes combinée à d'autres troubles augmenterait le risque de comportements suicidaires (Brent et al., 1993). En effet, la consommation de substances psychotropes peut agir en tant que déclencheur du geste suicidaire puisqu'elle diminue les inhibitions et augmente l'impulsivité. De même, la consommation de drogue peut avoir un effet dépresseur et favoriser les idéations suicidaires qui précèdent les comportements (Goldstein et al., 2005).

L'abus de substances psychotropes est fréquent chez les adolescents délinquants (DeMatteo et Marczyk, 2005; Goldstein et al., 2005). Chagnon et al. (2001) rapportent que les adolescents hébergés en centres de réadaptation et qui présentent des comportements suicidaires ont consommé de l'alcool plus fréquemment au cours de la dernière année comparativement à ceux qui ne présentent pas de tels comportements. L'intoxication aux drogues peut être un facteur précipitant du geste suicidaire dans $17 \%$ à $51 \%$ des cas des adolescents décédés par suicide dans des établissements pour adolescents (Chagnon et al., 2001). Dans l'étude de Hayes (2004) s'intéressant aux suicides chez les adolescents en détention, $87,9 \%$ des adolescents décédés par suicide lors de leur détention présentaient une histoire d'abus de substances. Pour d'autres (Howard et al., 2003), ce sont plutôt 26\% des cas de suicide chez les adolescents en détention qui sont associés à une consommation abusive de drogues et/ou d'alcool. Au-delà de ces divergences, l'abus de substances psychotropes est très prévalent chez les adolescents qui se suicident dans un contexte de détention. Dans une étude effectuée chez les adolescents incarcérés (Domalanta et al., 2003), $43 \%$ des garçons et $36 \%$ des filles avaient un problème d'abus d'alcool; $27 \%$ des garçons et des filles avaient un problème d'abus de substances psychotropes (Goldstein et al., 2005; Hart et al., 2007). En outre, l'abus de substances psychotropes et l'intoxication peuvent en soi exposer davantage les adolescents à des situations difficiles et ainsi interférer avec l'instauration de mécanismes d'adaptation efficaces, contribuant indirectement au risque suicidaire (Ruchkin et al., 2003).

\section{Caractéristiques individuelles et de personnalité}

Outre les troubles psychiatriques, les caractéristiques personnelles des adolescents délinquants augmentent leur risque de présenter des com- 
portements suicidaires (Putnins, 2005; Roberts et Bender, 2006). Plus spécifiquement, l'hostilité (Connor et Rueter, 2006), l'irritabilité (Beautrais, 2003a; Conner et al., 2004), la recherche de nouveauté (Beautrais, 2003a; Fergusson et al., 2003), la témérité, les comportements de prise de risque (Beautrais, 2003a; Vermeiren et al., 2003) et les comportements violents (Apter et al., 1988; Cleary, 2000; Marttunen et Pelkonen, 2000; American Academy of Child and Adolescent Psychiatry, 2001 ; Beautrais, 2003a ; Zlotnick et al., 2003) sont associés au risque de comportements suicidaires. Soulignons que ces mêmes caractéristiques sont utilisées pour décrire les adolescents délinquants et présentant des troubles des conduites (APA, 1994), ou pris en charge par le système judiciaire.

La violence et l'agressivité manifestées dès le jeune âge sont caractéristiques des adolescents délinquants (Lahey et al., 1999; Christoffersen et al., 2007; Goldstein et al., 2005). Ces mêmes éléments sont souvent en cause dans la prise en charge de ces jeunes par le système judiciaire. Les jeunes devenus délinquants ont souvent été décrits dès l'enfance comme ayant un tempérament "difficile», négatif, anxieux, irritable, colérique, agressifs et impulsifs (Holmes et al., 2001). Plusieurs études ont établi l'association entre la violence envers autrui et le risque de présenter des comportements suicidaires (Apter et al., 1989; Plutchik et Van Praag, 1990; Apter et al., 1993 ; Plutchik, 1995; Plutchik et al., 1995; Horesh et al., 1997; Chagnon et al., 2001; Daigle et Côté, 2006). L'association inverse est aussi vraie: les adolescents suicidaires démontrent plus de violence envers les autres que ceux qui ne sont pas suicidaires (Zlotnick et al., 2003).

Les recherches effectuées auprès d'adolescents délinquants établissent aussi l'association entre agressivité et suicide (Chowanec et al., 1991 ; Chagnon et al., 2001; Hukkanen et al., 2003; Wasserman et McReynolds, 2006; Brent et Bridge, 2007). Selon Plutchik et al. (1989) bien que l'agressivité manifestée contre soi et celle envers autrui possèdent des caractéristiques communes, il serait possible de départager ce qui différencie le recours à l'une ou à l'autre.

L'impulsivité est associée à la fois au risque de violence (Apter et al., 1993; DeMatteo et Marczyk, 2005) et au risque de suicide (Apter et al., 1993; Battle et al., 1993; Chagnon et al., 2001; Conner et al., 2004). Les patients hébergés en établissement psychiatrique qui ont commis une tentative de suicide ou qui présentent des idéations suicidaires démontreraient une plus grande impulsivité que les patients psychiatriques ne 
présentant pas de tels comportements. Hills et al. (2005) émettent l'hypothèse que l'impulsivité représente une caractéristique commune entre troubles externalisés et comportements suicidaires.

L'impulsivité apparaît comme un facteur de risque important des conduites suicidaires chez les jeunes délinquants (Rohde, Mace et al., 1997; Chagnon et al., 2001; Sanislow et al., 2003; Roberts et Bender, 2006; Brent et Bridge, 2007). Selon Apter et ses collègues (1995) les jeunes présentant des troubles des conduites seraient plus susceptibles d'avoir des comportements suicidaires de façon impulsive plutôt qu'en vertu d'un désir de mourir planifié, associé à la dépression majeure. Inversement, faire preuve de contrôle de son impulsivité constitue un facteur de protection en regard des comportements suicidaires; or, les jeunes en centres de réadaptation ont moins de contrôle en regard de leurs conduites impulsives que les adolescents qui n'y résident pas (Pronovost et al., 2003). Le contrôle de soi des adolescents délinquants est généralement plus déficient que chez ceux ne présentant pas cette problématique (Marcotte et al., 2002). Des lacunes ou un manque de confiance dans ses habiletés de résolution de problèmes (American Academy of Child and Adolescent Psychiatry, 2001 ; Esposito et Clum, 2002; Speckens et Hawton, 2005), une moins bonne gestion de la colère et de la tristesse ainsi que la réaction aux événements difficiles en se blâmant ou en étant agressif seraient des facteurs de risque de suicide (Chagnon et al., 2001; Chagnon et al., 2007). Les adolescents délinquants de l'étude de Hart et al. (2007) réagissaient plus souvent de façon agressive à la honte, témoignant possiblement de leur difficulté à gérer les émotions négatives.

Le sexe est un facteur qui a été étudié quant au risque de comportements suicidaires chez les adolescents. En général, les adolescentes seraient deux fois plus nombreuses à tenter de se suicider (Langlois et Morrison, 2002) alors que les adolescents décèdent plus par suicide que ces dernières (Gagné et St-Laurent, 2009). Néanmoins, en ce qui concerne le risque suicidaire chez les adolescents délinquants, à notre connaissance aucune recherche n'a porté spécifiquement sur ce sujet. Les résultats de l'étude de Pronovost et Leclerc (2002) chez les adolescents placés en centres jeunesse révèlent plus d'événements suicidaires chez les filles, mais ne permettent pas de conclure à une différence selon le genre chez les adolescents pris en charge spécifiquement en raison de leurs activités délinquantes (jeunes contrevenants). 


\section{Facteurs sociaux}

Famille

L'influence de la famille peut s'exercer de plusieurs façons sur les comportements suicidaires des adolescents. D'une part, la présence d'histoires de suicides dans la famille prédisposerait les adolescents à présenter des conduites suicidaires (Brent et al., 1996; Beautrais, 2000; American Academy of Child and Adolescent Psychiatry, 2001; Beautrais, 2001, 2003a; Fergusson et al., 2003; Portzky et al., 2005; Renaud et al., 2008). Plusieurs explications sont possibles pour comprendre cette association: les conséquences d'un suicide sur l'organisation et la dynamique familiale peuvent en soi prédisposer les jeunes à souffrir affectivement et à envisager le suicide; le suicide est fortement associé à la psychopathologie qui semble aussi être surreprésentée dans ces familles, tel qu'expliqué ci-après.

D'autre part, la psychopathologie des parents pourrait aussi augmenter le risque de suicide pour les adolescents (Brent et al., 1999; King et al., 2001 ; Flouri et Buchanan, 2002; Portzky et al., 2005). Ainsi, des parents qui abusent de substances psychotropes, des mères déprimées, des pères antisociaux, violents et abusifs mettraient aussi les adolescents plus à risque de présenter des comportements suicidaires (Brent et al., 1994; Brent et al., 1996; Renaud et al., 1999; Beautrais, 2000; American Academy of Child and Adolescent Psychiatry, 2001; Brent et Bridge, 2007).

En plus de contribuer au risque suicidaire, les pratiques parentales et le climat familial sont fortement associés aux comportements délinquants (Patterson et al., 1989; Farrington, 1994; Butler et al., 2007). En effet, les enfants issus de familles instables et conflictuelles présentent plus de risques de délinquance que ceux issus de familles unies et paisibles où les parents offrent une supervision adéquate et constante (Patterson et al., 1989). Les enfants ayant grandi dans des familles caractérisées par la violence familiale, où les parents abusent de substances psychotropes, présentent des troubles psychiatriques et n'occupent pas d'emploi stable seraient plus à risque de développer des problématiques de délinquance (DeMatteo et Marczyk, 2005; Christoffersen et al., 2007). Le fait de vivre dans une famille où les parents sont séparés augmenterait le risque de présenter des comportements suicidaires à l'adolescence (Flouri et Buchanan, 2002; Flouri, 2005). De même, avoir des parents 
ayant des difficultés légales (Brent et al., 1994) ou impliqués dans le milieu criminel (Brent et Bridge, 2007) entraînait des risques plus élevés de comportements suicidaires et de délinquance chez leurs adolescents (Lahey et al., 1999; Alltucker et al., 2006).

Les facteurs de risque parentaux (psychopathologie, conduites suicidaires, criminalité) peuvent en effet compromettre les habiletés parentales et l'exercice de leur rôle de référence stable auprès de leurs adolescents. D'autres indices appuient cette hypothèse selon laquelle la supervision et le soutien émotionnel des parents sont essentiels à l'adolescence et qu'ils ont une influence sur les comportements suicidaires (Flouri et Buchanan, 2002; Perkins et Hartless, 2002 ; Flouri, 2005 ; Kidd et al., 2006). Les adolescents délinquants qui présentent un profil suicidaire perçoivent leurs parents comme peu chaleureux et se sentent fréquemment rejetés par ces derniers (Ruchkin et al., 2003).

\section{Réseau social}

Des recherches qui se sont intéressées aux adolescents délinquants ont pu mettre de l'avant que ces jeunes présentent un réseau social déficient (Rohde, Seeley et al., 1997; DeMatteo et Marczyk, 2005). Les comportements hostiles, agressifs et violents de ces jeunes en font souvent des enfants non populaires à l'école, ce qui contribue en retour au développement de leurs comportements délinquants (Goldstein et al., 2005) et à l'affiliation à des pairs présentant des comportements semblables (Holmes et al., 2001). De plus, le fait de se sentir rejeté par ses pairs peut entraîner du désespoir, de la dépression, des idéations et des comportements suicidaires (Prinstein et al., 2000). Deux études observent chez les adolescents affiliés à un gang plus d'idéations suicidaires et de tentatives de suicide (Morris et al., 1995 ; Prinstein et al., 2000). Par ailleurs, l'affiliation à un groupe de pairs délinquants, bien qu'elle augmente la vulnérabilité du jeune au suicide (Beautrais, 2003a; Fergusson et al., 2003), peut dans certains cas constituer le seul réseau de soutien de l'adolescent délinquant. Il est primordial que les intervenants demeurent vigilants face au risque suicidaire pouvant être présenté par un jeune à qui on décide d'interdire tout contact avec des pairs délinquants, même si cela est fait dans un but de réhabilitation. 


\section{Facteurs résidentiels}

Milieu de vie et voisinage

Provenir d'un milieu désavantagé sur le plan socio-économique constituerait un facteur de risque de comportements suicidaires (Beautrais, 2000, 2003a ; Brent et Bridge, 2007) et délinquants (Farrington, 1994; DeMatteo et Marczyk, 2005). Ce risque pourrait agir de façon indirecte. En effet, des difficultés d'ordre économique et l'absence de ressources nécessaires pourraient entraver l'exercice du rôle parental. De façon plus directe, la présence de facteurs de risque, notamment l'accès à des armes à feu ou d'autres moyens létaux, peut augmenter le risque de suicide chez les adolescents (Rohde, Seeley et al., 1997; Wasserman et McReynolds, 2006).

\section{École}

Une faible motivation scolaire, des attitudes négatives face à l'école et des difficultés scolaires représentent des facteurs de risque de comportements suicidaires chez les adolescents (American Academy of Child and Adolescent Psychiatry, 2001; Flouri et Buchanan, 2002; Portzky et al., 2005; Hart et al., 2007). Quitter l'école et ne pas atteindre des qualifications minimales placerait les adolescents plus à risque de présenter des comportements suicidaires (Rohde, Seeley et al., 1997; Beautrais, 2003a, 2003b; Fergusson et al., 2003; Brent et Bridge, 2007).

Milieu résidentiel pour jeunes contrevenants

Vivre dans un établissement pour délinquants juvéniles constituerait en soi un facteur de risque (Pena et Caine, 2006; Roberts et Bender, 2006). L'étude de Memory (1989) établit que séjourner dans un établissement de détention pour mineurs augmente de 4,6 fois le risque de suicide. Afin d'expliquer ce taux élevé de suicide dans les établissements pour délinquants juvéniles, certains chercheurs (Howard et al., 2003) ont souligné que les tentatives de suicide reflètent le niveau de détresse et de difficulté de l'adolescent contrevenant. En ce sens, la tentative de suicide, tout comme l'ordonnance d'hébergement, sont considérées comme conséquences d'un état de détresse et de désorganisation envahissant. De même, il importe de ne pas perdre de vue que ces institutions hébergent des adolescents déjà à risque de suicide et de comportements suicidaires par les problèmes qu'ils présentent au moment de leur admission (Roberts et Bender, 2006). 


\section{Conclusion de l'étude des facteurs de risque}

En somme, la revue des études sur le suicide montre que des facteurs communs contribuent à la délinquance et au risque suicidaire chez les jeunes contrevenants. De même, les impacts négatifs des comportements délinquants manifestés par les jeunes augmentent de façon indirecte, par le stress qu'ils génèrent, leur vulnérabilité et leur risque suicidaire. Afin de mieux comprendre comment agissent les divers facteurs associés à la fois à la délinquance et au risque suicidaire, nous proposons de les conceptualiser à l'intérieur d'un modèle explicatif qui puisse prendre en compte leurs relations causales. Une telle modélisation permet de mieux cerner, dans une trajectoire de vie, les facteurs de risque et les opportunités de prévention. La section qui suit présente un modèle explicatif de la délinquance et du risque suicidaire.

\section{Un modèle explicatif}

À partir de l'étude des facteurs de risque tels qu'ils ont été présentés dans la section précédente, il est possible d'en dégager un modèle explicatif qui permet de les intégrer au cœur même de la dynamique $\mathrm{du}$ jeune contrevenant. Ce dernier constitue le noyau autour duquel gravitent les facteurs susceptibles de contribuer et de moduler son risque suicidaire. Ce modèle permet de mieux comprendre comment l'interaction entre les facteurs de risque peut, dans une perspective temporelle, ajouter au risque de suicide. De même, chacun des facteurs de risque proximaux ou lointains présentés constitue une possibilité d'intervention ou différentes «portes» par lesquelles la prévention des comportements suicidaires et du suicide peut être conceptualisée. Le modèle explicatif proposé à la figure 1 (page suivante) illustre la dynamique interactive des facteurs de risque du suicide chez les jeunes délinquants.

Ce modèle interactif suggère que des facteurs antérieurs aux comportements délinquants (facteurs liés aux événements de vie, aux conditions sociales et familiales, aux facteurs résidentiels, etc.), en conjonction avec des éléments reliés à la personnalité et des facteurs contemporains à la délinquance (commission du délit, prise en charge et conséquences socio-judiciaires), interagissent de façon concomitante en créant une dynamique d'aggravation et de chronicisation au fil du temps. Comme conséquence de cette dynamique, l'adolescent est plus à risque de souffrir de dépression, de vivre du désespoir et/ou de 
l'anxiété face à l'impossibilité de trouver des solutions adéquates à sa situation, ce qui augmente le risque de comportements suicidaires et ultimement de suicide.

F I G URE 1

Modèle interactif des facteurs associés au risque suicidaire chez les jeunes contrevenants

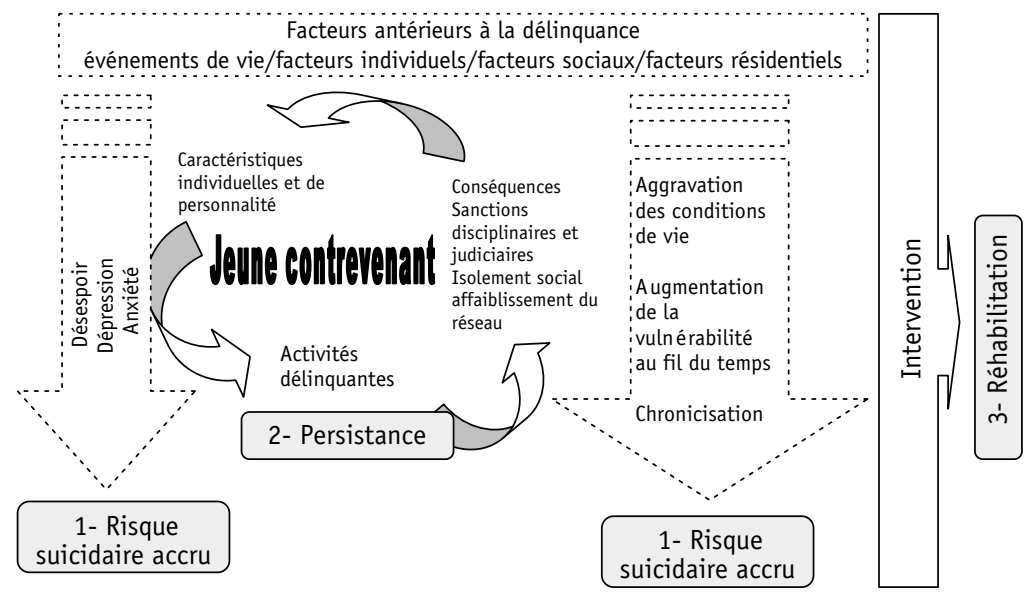

\section{Illustration du modèle par une histoire d'adolescent contrevenant}

Afin d'illustrer le contexte dans lequel peuvent prendre place les idéations et les conduites suicidaires chez les adolescents pris en charge par la Loi sur le système de justice pénale pour adolescents, nous avons choisi l'histoire d'Olivier (vignette clinique, page suivante). Il s'agit d'un jeune homme de 18 ans actuellement hébergé en garde fermée dans un centre de réadaptation de la région de Montréal. Olivier a connu deux épisodes au cours desquels il a expérimenté des idéations et eu des conduites suicidaires. À l'heure actuelle, Olivier exprime le désir de se réhabiliter.

En se basant sur le modèle interactif du comportement suicidaire (figure 1) pour l'étude de l'histoire d'Olivier, il est possible de constater que c'est dans des moments où l'adolescent n'avait plus l'espoir de pouvoir améliorer sa situation qu'il entretenait des idées suicidaires et passait à l'acte. Dans une perspective préventive, il est possible de constater que l'identification des facteurs de risque mis en lumière dans 


\section{F I G U R 2}

\section{Vignette clinique - Olivier}

Depuis sa naissance, Olivier a connu de près le milieu criminel. Son père est membre d'un groupe criminel organisé et sa mère vend et consomme de la drogue. L'adolescent a été abusé sexuellement par deux membres de la famille qui devaient prendre soin de lui en l'absence de sa mère alors qu'il était âgé de 8 ans. Au cours de cette même période, il aurait agressé et menacé physiquement sa mère. Cette dernière a alors alerté la DPJ et un placement en famille d'accueil a suivi.

C'est à l'âge de 11 ans qu'Olivier a connu son premier épisode suicidaire. À cette époque, Olivier trouvait extrêmement difficile d'être séparé de ses parents par son placement. Olivier a ensuite connu de multiples allers-retours entre des placements en centres de réadaptation et des fugues. Depuis l'âge de 13 ans, il vend et consomme des stupéfiants lors de ses fugues. C'est lors d'une ordonnance de placement en centre de réadaptation qu'Olivier a connu une seconde période suicidaire. Olivier nous explique qu'à ce moment, il avait perdu espoir de s'en sortir et voyait tout en noir à cause de sa famille, ne voyant pas comment il pourrait aller mieux et quitter le milieu criminel puisque tous les membres de sa famille en faisaient partie.

À sa demande, Olivier voit maintenant un psychologue une fois par semaine. Il dit que cela lui fait beaucoup de bien de voir ce qu'il se passe pour lui. Il tente de faire le deuil de ses parents parce qu'il ne croit pas pouvoir s'en sortir s'il continue de les fréquenter régulièrement. Pour lui, ses parents ne sont pas un modèle à suivre.

le modèle permet l'ouverture de «portes» pour l'intervention. Plus précisément, dès son tout jeune âge, Olivier présentait des facteurs de risque, tant pour l'établissement d'une dynamique délinquante que pour la mise en place d'un risque suicidaire. Pensons notamment aux activités criminelles dans lesquelles ses parents sont impliqués, à la consommation de drogue de la part de sa mère et aux agressions sexuelles dont il a été victime qui constituent des facteurs de risque liés aux facteurs sociaux et à des événements de vie traumatiques. La prévention pouvant être mise en place sur ce plan - prévention primaire ${ }^{2}$ - devrait être élaborée dans le but d'éviter les abus, les traumas, ainsi que des conditions de vie défavorables pour les enfants, ce qui s'inscrit d'ailleurs de pair avec les efforts déployés en ce sens par la Loi sur la protection de la

2. Selon l'Organisation mondiale de la Santé (OMS), la prévention primaire comprend les «mesures applicables à une maladie ou groupe de maladies pour en bloquer les causes avant qu'elles n'agissent sur l'homme; en d'autres termes, pour empêcher la survenue de la maladie». La prévention secondaire concerne les «mesures destinées à interrompre un processus morbide en cours pour prévenir de futures complications et séquelles, limiter les incapacités et éviter le décès». La prévention tertiaire comprend les «mesures visant à permettre aux personnes handicapées de recouvrer leurs fonctions initiales ou d'utiliser au maximum les capacités qui leur restent; la réadaptation comprend à la fois des interventions individuelles et des actions sur l'environnement.» (Définitions tirées du site internet de l'OMS - www.who.int/whr/2001/chapter3/fr/index3.html - consulté le 16 mars 2010.) 
jeunesse. Une seconde porte d'entrée en ce qui concerne la prévention primaire des comportements suicidaires auprès d'Olivier est d'intervenir dans le domaine de ses caractéristiques individuelles, tant sur le plan de la psychopathologie que de la personnalité. En somme, ce jeune homme est impulsif, a tendance à utiliser l'agressivité et la violence comme moyens de régler les conflits et a entre autres souffert de troubles dépressifs à plus d'une reprise dans sa vie. En prévention primaire, il importe que les intervenants soient vigilants dans la détection de ces indices, mais aussi qu'ils puissent orienter le jeune vers des ressources ou des programmes adéquats. Dans le cas d'Olivier, il pourrait être approprié de l'aider à mieux tolérer les délais et de lui apprendre à régler les problèmes par d'autres moyens que par la violence. À cette fin, la participation à un groupe de thérapie sur l'apprentissage de la gestion des conflits pourrait s'avérer utile. En ce qui concerne la prévention secondaire, un suivi psychothérapeutique serait nécessaire afin de traiter la dépression et de le soutenir dans la définition de ses objectifs de vie. En effet, Olivier exprime fréquemment ressentir un grand désespoir lorsqu'il pense à ses parents, sur lesquels il ne peut s'appuyer pour se construire une vie «légale» puisque ces derniers constituent plutôt des modèles délinquants. Une troisième porte d'intervention préventive, qu'il est possible de qualifier de prévention tertiaire, concerne les conséquences qu'entraînent les activités délinquantes. En conséquence, les intervenants devraient redoubler de vigilance lorsqu'un jeune en est au début d'une ordonnance d'hébergement et dans les périodes d'hébergement en attente d'une décision du tribunal. Ces «conséquences» de la délinquance constituent des périodes très stressantes qui peuvent susciter du désespoir et favoriser l'émergence de comportements suicidaires. Pensons notamment aux facteurs résidentiels propres aux milieux d'intervention où vivre dans un lieu où les portes sont verrouillées et en chambre individuelle semble augmenter le risque suicidaire en exacerbant le sentiment d'isolement du jeune déjà perturbé par son placement. Chez Olivier, c'est d'ailleurs lors du début d'un placement qu'il nous raconte s'être senti découragé et avoir commis des actes suicidaires. En ce sens, il est primordial que le milieu d'intervention puisse bien évaluer le risque suicidaire et mette en place des interventions adaptées aux difficultés éprouvées par le jeune. Par exemple, tenant compte des facteurs résidentiels, il pourrait être plus adéquat de ne pas réagir aux idéations suicidaires en isolant davantage l'adolescent qui les exprime. L'isolement pourrait avoir comme effet d'inhiber l'expression des idéa- 
tions suicidaires ou encore d'exacerber le sentiment de désespoir. Sous cette même catégorie «conséquences», nous notons dans le modèle interactif l'épuisement du réseau de soutien et l'affaiblissement des liens sociaux. Le moment de la prise en charge pourrait en ce sens constituer une porte d'entrée vers le développement de relations sociales positives en accompagnant l'adolescent dans la redéfinition de son réseau social. Lapproche temporelle de la «crise suicidaire» proposée par Morasz (2008) est appropriée puisqu'elle permet de prendre en compte les signes précurseurs et les facteurs de risque dans la prise en charge.

En plus d'identifier les diverses avenues préventives permises par le modèle interactif, nous visons la compréhension du processus qui permet d'expliquer que certains jeunes exposés à ces facteurs présenteront des comportements suicidaires alors que d'autres n'en présenteront pas. Nous émettons donc l'hypothèse de trois devenirs possibles pour le jeune pris au centre de cette dynamique de facteurs de risque. En premier lieu, un jeune contrevenant pris au centre de ce cercle vicieux où les conséquences de ses actions délinquantes ne font qu'envenimer sa situation (1) peut n'y voir aucune issue possible, alimentant ses affects dépressifs et son désespoir, c'est ce que nous appelons «aggravation» ou «chronicisation». C'est en réponse à cette impuissance que nous croyons que certains jeunes présentent un risque accru de conduites suicidaires. Il est aussi possible que le jeune contrevenant (2) persiste dans les comportements délinquants qu'il connaît déjà, c'est ce que nous nommons «persistance de la délinquance». À l'inverse, le jeune contrevenant peut (3) se réhabiliter et ne plus agir de façon délinquante, il peut s'engager sur la voie que ses intervenants souhaitent qu'il prenne, répondant ainsi aux énergies qu'ils déploient en ce sens, c'est ce que nous nommons la «réhabilitation». Le modèle intègre la réhabilitation à la suite de l'intervention pouvant se faire à tous les niveaux de risque, tel que cela a été expliqué précédemment par l'ouverture de «portes» pour la prévention. Il importe ici de ne pas omettre de considérer ces adolescents à risque qui se «réhabilitent» dans la société en raison d'une grande résilience, soit «la capacité de l'être humain à résister, rebondir et se reconstruire malgré la survenue d'un ou de plusieurs traumatismes» (Tychey et Lighezzolo, 2004). La résilience chez les adolescents délinquants s'expliquerait surtout par des facteurs personnels tels le contrôle de soi et la maturité (Born et al., 1997). Les trois devenirs mis en lumière par le modèle constituent trois types de réactions que peuvent avoir les jeunes contrevenants qui arborent les facteurs de risque 
que nous avons exposés précédemment. La figure 1 présentée précédemment intègre d'ailleurs ces trois devenirs (1- risque suicidaire accru; 2- persistance; 3- réhabilitation).

Il semble qu'Olivier ait aujourd'hui l'espoir de pouvoir aller mieux et de ne pas poursuivre ses activités délinquantes une fois sa sentence terminée. En ce sens, le cas d'Olivier illustre la sortie «réhabilitation» du modèle théorique proposé.

En conclusion, en adoptant un modèle interactif qui propose une explication fondée sur l'intégration des conditions antérieures, des conditions concomitantes et des conséquences de la délinquance, il est possible de comprendre le suicide comme la résultante d'un processus alliant stress et vulnérabilité, tel que l'illustre la figure 1. En se basant sur le modèle interactif proposé, la prévention devrait cibler différentes «portes» correspondant à ces groupes de facteurs de risque, comme le cas d'Olivier a permis de le démontrer. Le modèle interactif intégré a en outre permis de déceler trois devenirs du jeune délinquant - l'aggravation du risque suicidaire, la persistance dans la délinquance et la réhabilitation - qui ont été intégrés au modèle. La recherche devra maintenant mettre au jour et étudier les facteurs spécifiques pouvant mener à ces trois devenirs chez des individus qui présentent par ailleurs des facteurs de risque similaires. Il s'agira ainsi de prendre en considération à la fois les facteurs intrapsychiques (mécanismes de défense utilisés, registre psychique) et les facteurs relevant de l'environnement de l'individu (personnes ressources, organismes de soutien, etc.) dans la compréhension de la dynamique du processus suicidaire. De même, nous croyons important de s'attarder aux diverses conduites à risque qui ne sont pas considérées comme conduites suicidaires, mais qui interrogent le désir de vivre des adolescents. Dans cette catégorie de conduites figurent les activités délinquantes qui mettent la vie des protagonistes en danger, mais aussi les comportements de recherche de sensations extrêmes (conduite dangereuse, consommation de drogues dures, comportements sexuels à risque, etc.). 


\section{Références}

Abram, K. M., Choe, J. Y., Washburn, J. J., Teplin, L. A., King, D. C., \& Dulcan, M. K. (2008). Suicidal Ideation and Behaviors Among Youths in Juvenile Detention. Journal of the American Academy of Child $\mathcal{O}$ Adolescent Psychiatry, 47 (3), 291-300.

Alessi, N. E., McManus, M., Brickman, A., \& Grapentine, L. (1984). Suicidal behavior among serious juvenile offenders. American Journal of Psychiatry, 141 (2), 286-287.

Alltucker, K. W., Bullis, M., Close, D., \& Yovanoff, P. (2006). Different Pathways to Juvenile Delinquency: Characteristics of Early and Late Starters in a Sample of Previously Incarcerated Youth. Journal of Child and Family Studies, 15 (4), 479-492.

American Academy of Child and Adolescent Psychiatry (2001). Practice Parameter for the Assessment and Treatment of Children and Adolescent With Suicidal Behavior. Journal of the American Academy of Child and Adolescent Psychiatry, 40 (7 [suppl.]), 24S-51S.

Apter, A., Bleich, A., Plutchik, R., Mendelsohn, S., \& Tyano, S. (1988). Suicidal behavior, depression, and conduct disorder in hospitalized adolescents. Journal of the American Academy of Child $\mathcal{F}$ Adolescent Psychiatry, 27 (6), 696699.

Apter, A., Gothelf, D., Orbach, I., Weizman, R., \& Ratzoni, G. (1995). Correlation of suicidal and violent behavior in different diagnostic categories in hospitalized adolescent patients. Journal of the American Academy of Child $\mathbb{F}$ Adolescent Psychiatry, 34 (7), 912-918.

Apter, A., Plutchik, R., Sevy, S., Korn, M. L., \& Brown, S. L. (1989). Defense mechanisms in risk of suicide and risk of violence. American Journal of Psychiatry, 146 (8), 1027-1031.

Apter, A., Plutchik, R., \& Van Praag, H. M. (1993). Anxiety, impulsivity and depressed mood in relation to suicidal and violent behavior. Acta Psychiatrica Scandinavica, 87 (1), 1-5.

Battle, A. O., Battle, M. V., \& Tolley, E. A. (1993). Potential for suicide and aggression in delinquents at Juvenile Court in a southern city. Suicide and Life-Threatening Behavior, 23 (3), 230-244.

Beautrais, A. L. (2000). Risk factors for suicide and attempted suicide among young people. Australian and New Zealand Journal of Psychiatry, 34 (3), 420-436.

Beautrais, A. L. (2001). Child and young adolescent suicide in New Zealand. Australian and New Zealand Journal of Psychiatry, 35 (5), 647-653.

Beautrais, A. L. (2002). Gender issues in youth suicidal behaviour. Emergency Medicine, 14, 35-42.

Beautrais, A. L. (2003a). Life course factors associated with suicidal behaviors in young people. American Behavioral Scientist. Special Issue: Suicide in Youth, 46 (9), 1137-1156.

Beautrais, A. L. (2003b). Suicide and Serious Suicide Attempts in Youth: A Multiple-Group Comparison Study. American Journal of Psychiatry, 160 (6), 1093-1099. 
Born, M., Chevalier, V., \& Humblet, I. (1997). Resilience, desistance and delinquent career of adolescent offenders. Journal of Adolescence, 20, 679-694.

Boyer, R., Saint-Laurent, D., Préville, M., Légaré, G., Massé, R., \& Poulin, C. (2000). Idées suicidaires et parasuicides. In Enquête sociale et de santé 1998 (355-366). Québec: Institut de la statistique du Québec.

Brent, D. A., Baugher, M., Bridge, J., Chen, T., \& Chiappetta, L. (1999). Ageand sex-related risk factors for adolescent suicide. Journal of the American Academy of Child $\mathfrak{O}$ Adolescent Psychiatry, 38 (12), 1497-1505.

Brent, D. A., Bridge, J., Johnson, B. A., \& Connolly, J. (1996). Suicidal behavior runs in families: A controlled family study of adolescent suicide victims. Archives of General Psychiatry, 53 (12), 1145-1152.

Brent, D. A., \& Bridge, J. A. (2007). Delinquent Accounts: Does Delinquency Account for Suicidal Behavior? Journal of Adolescent Health, 40 (3), 204-205.

Brent, D. A., Perper, J. A., Moritz, G., Allman, C., et al. (1993). Psychiatric risk factors for adolescent suicide: A case-control study. Journal of the American Academy of Child $\mathfrak{W}$ Adolescent Psychiatry, 32 (3), 521-529.

Brent, D. A., Perper, J. A., Moritz, G., Liotus, L., Schweers, J., Balach, L., \& Roth, C. (1994). Familial risk factors for adolescent suicide: A case-control study. Acta Psychiatrica Scandinavica, 89 (1), 52-58.

Butler, S., Fearon, P., Atkinson, L., \& Parker, K. (2007). Testing an interactive model of symptom severity in conduct disordered youth: Family relationships, antisocial cognitions, and social-contextual risk. Criminal Justice and Behavior, 34 (6), 721-738.

Chagnon, F. (2000). Les événements stressants, les mécanismes d'adaptation et le suicide chez les adolescents admis en centres de réadaptation. Montréal: Université du Québec à Montréal.

Chagnon, F., Houle, J., Marcoux, I., \& Renaud, J. (2007). Control-group study of an intervention training program for youth suicide prevention. Suicide and Life-Threatening Behavior, 37 (2), 135-144.

Chagnon, F., Renaud, J., \& Farand, L. (2001). Suicide et délinquance juvénile: phénomènes distincts ou manifestations d'une même problématique? Criminologie, 34 (2), 85-102.

Chapman, J., F., \& Ford, J. D. (2008). Relationships Between Suicide Risk, Traumatic Experiences, and Substance Use Among Juvenile Detainees. Archives of Suicide Research, 12, 50-61.

Chowanec, G. D., Josephson, A. M., Coleman, C., \& Davis, H. (1991). Selfharming behavior in incarcerated male delinquent adolescents. Journal of the American Academy of Child $\mho \sim$ Adolescent Psychiatry, 30 (2), 202-207.

Christoffersen, M. N., Soothill, K., \& Francis, B. (2007). Violent life events and social disadvantage: A systematic study of the social background of various kinds of lethal violence, other violent crime, suicide, and suicide attempts. Journal of Scandinavian Studies in Criminology and Crime Prevention, 8 (2), 157184.

Cleary, S. D. (2000). Adolescent victimization and associated suicidal and violent behaviors. Adolescence, 35 (140), 671-682. 
Conner, K. R., Meldrum, S., Wieczorek, W. F., Duberstein, P. R., \& Welte, J. W. (2004). The Association of Irritability and Impulsivity with Suicidal Ideation Among 15- to 20-year-old Males. Suicide and Life-Threatening Behavior, 34 (4), 363-373.

Connor, J. J., \& Rueter, M. A. (2006). Parent-Child Relationships as Systems of Support or Risk for Adolescent Suicidality. Journal of Family Psychology, 20 (1), 143-155.

Daigle, M. S., \& Côté, G. (2006). Non-Fatal Suicide-Related Behavior among Inmates: Testing for Gender and Type Differences. Suicide and Life-Threatening Behavior, 36 (6), 670-681.

Davis, D. L., Bean, G. J., Schumacher, J. E., \& Stringer, T. L. (1991). Prevalence of emotional disorders in a juvenile justice institutional population. American Journal of Forensic Psychology, 9 (1), 5-17.

DeMatteo, D., \& Marczyk, G. (2005). Risk Factors, Protective Factors, and the Prevention of Antisocial Behavior Among Juveniles. In K. Heilbrun, N. Goldstein \& R. Redding (Eds.), Juvenile delinquency: Prevention, assessment, and intervention (19-44). New York: Oxford University Press.

Dembo, R., Williams, L., Wish, E. D., Berry, E., Getreu, A., Washburn, M., \& Schmeidler, J. (1990). Examination of the Relationships Among Drug Use, Emotional/Psychological Problems, and Crime Among Youths Entering a Juvenile Detention Center. The International Journal of Addictions, 25 (11), 13011340.

Diagnostic and statistical manual of mental disorders (4th ed.). (1994). Washington, DC: US : American Psychiatric Publishing, Inc, 886.

Domalanta, D. D., Risser, W. L., Roberts, R. E., \& Risser, J. M. H. (2003). Prevalence of depression and other psychiatric disorders among incarcerated youths. Journal of the American Academy of Child $\mho$ Adolescent Psychiatry, 42 (4), 477-484.

Dube, S. R., Anda, R. F., Felitti, V. J., Chapman, D. P., Williamson, D. F., \& Giles, W. H. (2001). Childhood abuse, household dysfunction, and the risk of attempted suicide throughout the life span: Findings from the adverse childhood experiences study. JAMA : Journal of the American Medical Association, 286 (24), 3089-3096.

Esposito, C. L., \& Clum, G. A. (2002). Psychiatric symptoms and their relationship to suicidal ideation in a high-risk adolescent community sample. Journal of the American Academy of Child or Adolescent Psychiatry, 41 (1), 44-51.

Farand, L., Chagnon, F., Renaud, J., \& Rivard, M. (2004). Completed Suicides Among Quebec Adolescents Involved With Juvenile Justice and Child Welfare Services. Suicide and Life-Threatening Behavior, 34 (1), 24-35.

Farrington, D. P. (1994). The Twelfth Jack Tizard Memorial Lecture. The Development of Offending and Antisocial Bebaviour from Childhood: Key Findings from the Cambridge Study in Delinquent Development. Paper presented at the ACPP 2nd European Conference.

Fergusson, D. M., Beautrais, A. L., \& Horwood, L. J. (2003). Vulnerability and resiliency to suicidal behaviours in young people. Psychological Medicine, 33 (1), 61-73. 
Fergusson, D. M., Horwood, L. J., Ridder, E. M., \& Beautrais, A. L. (2005). Suicidal behaviour in adolescence and subsequent mental health outcomes in young adulthood. Psychological Medicine, 35 (7), 983-993.

Flouri, E. (2005). Psychological and Sociological Aspects of Parenting and Their Relation to Suicidal Behavior. Archives of Suicide Research, 9 (4), 373-383.

Flouri, E., \& Buchanan, A. (2002). The protective role of parental involvement in adolescent suicide. Crisis: The Journal of Crisis Intervention and Suicide Prevention, 23 (1), 17-22.

Fombonne, E. (1998). Suicidal behaviors in vulnerable adolescents: Time trends and their correlates. British Journal of Psychiatry, 173, 154-159.

Fombonne, E., Wostear, G., Cooper, V., Harrington, R., \& Rutter, M. (2001). The Maudsley long-term follow-up of child and adolescent depression: 2 . Suicidality, criminality and social dysfunction in adulthood. British Journal of Psychiatry, 179 (3), 218-223.

Gagné, M., \& St-Laurent, D. (2009). La mortalité par suicide au Québec: tendances et données récentes 1981-2007. Québec: Institut national de santé publique du Québec.

Gallagher, C. A., \& Dobrin, A. (2006). Deaths in juvenile justice residential facilities. Journal of Adolescent Health, 38 (6), 662-668.

Goldstein, N. E. S., Olubadewo, O., Redding, R. E., \& Lexcen, F. J. (2005). Mental Health Disorders: The Neglected Risk Factor in Juvenile Delinquency. In K. Heilbrun (Ed.), Juvenile delinquency: Prevention, assessment, and intervention (85-110). New York: Oxford University Press.

Hamel, D. (2001). Évolution des traumatismes au Québec de 1991 à 1999. Québec: Institut national de santé publique.

Harris, T. E., \& Lennings, C. J. (1993). Suicide and adolescence. International Journal of Offender Therapy and Comparative Criminology, 37 (3), 263-270.

Hart, J. L., O’Toole, S. K., Price-Sharps, J. L., \& Shaffer, T. W. (2007). The risk and protective factors of violent juvenile offending: An examination of gender differences. Youth Violence and Juvenile Justice, 5 (4), 367-384.

Hayes, L. M. (2005). Juvenile Suicide in Confinement in the United States: Results From a National Survey. Crisis: The Journal of Crisis Intervention and Suicide Prevention, 26 (3), 146-148.

Hills, A. L., Cox, B. J., McWilliams, L. A., \& Sareen, J. (2005). Suicide attempts and externalizing psychopathology in a nationally representative sample. Comprehensive Psychiatry, 46, 334-339.

Holmes, S. E., Slaughter, J. R., \& Kashani, J. (2001). Risk factors in childhood that lead to the development of conduct disorder and antisocial personality disorder. Child Psychiatry \& Human Development, 31 (3), 183-193.

Horesh, N., Rolnick, T., Iancut, I., Dannon, P., Lepkifker, E., Apter, A., \& Kotler, M. (1997). Anger, impulsivity and suicide risk. Psychotherapy and Psychosomatics, 66 (2), 92-96.

Howard, J., Lennings, C. J., \& Copeland, J. (2003). Suicidal behavior in a young offender population. Crisis: The Journal of Crisis Intervention and Suicide Prevention, 24 (3), 98-104. 
Hukkanen, R., Sourander, A., \& Bergroth, L. (2003). Suicidal ideation and behavior in children's homes. Nordic Journal of Psychiatry, 57 (2), 131-137.

Kempton, T., \& Forehand, R. L. (1992). Suicide attempts among juvenile delinquents: The contribution of mental health factors. Bebaviour Research and Therapy, 30 (5), 537-541.

Kidd, S., Henrich, C. C., Brookmeyer, K. A., Davidson, L., King, R. A., \& Shahar, G. (2006). The Social Context of Adolescent Suicide Attempts: Interactive Effects of Parent, Peer, and School Social Relations. Suicide and Life-Threatening Behavior, 36 (4), 386-395.

King, R. A., Schwab-Stone, M., Flisher, A. J., Greenwald, S., Kramer, R. A., Goodman, S. H., et al. (2001). Psychosocial and risk behavior correlates of youth suicide attempts and suicidal ideation. Journal of the American Academy of Child o Adolescent Psychiatry, 40 (7), 837-846.

Kotila, L., \& Lönnqvist, J. (1988). Adolescent suicide attempts: Sex differences predicting suicide. Acta Psychiatrica Scandinavica, 77 (3), 264-270.

Lahey, B. B., Waldman, I. D., \& McBurnett, K. (1999). The development of antisocial behavior: An integrative causal model. Journal of Child Psychology and Psychiatry, 40 (5), 669-682.

Langhinrichsen-Rohling, J., Arata, C., Bowers, D., O’Brien, N., \& Morgan, A. (2004). Suicidal Behavior, Negative Affect, Gender, and Self-Reported Delinquency in College Students. Suicide and Life-Threatening Behavior, 34 (3), 255-266.

Langlois, S., \& Morrison, P. (2002). Suicides et tentatives de suicide. Rapports sur la santé, 13 (2), 9-25. N 82-003-XPF au catalogue. Statistique Canada.

Lay, B., Ihle, W., Esser, G., \& Schmidt, M. H. (2005). Juvenile-episodic, Continued or Adult-onset Delinquency? European Journal of Criminology, 2 (1), 39-66.

Marcotte, G., Marcotte, D., \& Bouffard, T. (2002). The influence of familial support and dysfunctional attitudes on depression and delinquency in an adolescent population. European Journal of Psychology of Education, 17 (4), 363-376.

Marttunen, M., \& Pelkonen, M. (2000). Psychiatric risk factors for adolescent suicide--a review. Psychiatria Fennica, 31, 110-125.

McReynolds, L., S., Wasserman, G., A., DeComo, R., E., John, R., Keating, J., M., \& Nolen, S. (2008). Psychiatric Disorder in a Juvenile Assessment Center. Crime and Delinquency, 54 (2), 313-334.

Memory, J. M. (1989). Juvenile suicides in secure detention facilities: Correction of published rates. Death Studies, 13 (5), 455-463.

Morasz, L. (2008). La dynamique suicidaire. L. Morasz \& F. Danet (Eds), Comprendre et soigner la crise suicidaire (93-116) Paris: Dunod.

Morris, R. E., Harrison, E. A., Knox, G. W., Tromanhauser, E., \& et al. (1995). Health risk behavioral survey from 39 juvenile correctional facilities in the United States. Journal of Adolescent Health, 17 (6), 334-344.

Nolen, S., McReynolds, L. S., DeComo, R. E., John, R., Keating, J. M., \& Wasserman, G. A. (2008). Lifetime suicide attempts in Juvenile Assessment Center youth. Archives of Suicide Research, 12 (2), 111-123. 
Otzenberger, J. H. (2003). Personality characteristics relating to suicide risk of incarcerated adolescent males as measured by the Millon Adolescent Clinical Inventory and the Suicide Probability Scale. Dissertation Abstracts International: Section B: The Sciences and Engineering, 64 (6-B), 2934.

Patterson, G. R., DeBaryshe, B. D., \& Ramsey, E. (1989). A developmental perspective on antisocial behavior. American Psychologist. Special Issue: Children and their development: Knowledge base, research agenda, and social policy application, 44 (2), 329-335.

Pena, J. B., \& Caine, E. D. (2006). Screening as an Approach for Adolescent Suicide Prevention. Suicide and Life-Threatening Behavior, 36 (6), 614-637.

Penn, J. V., Esposito, C. L., Schaeffer, L. E., Fritz, G. K., \& Spirito, A. (2003). Suicide attempts and self-mutilative behavior in a juvenile correctional facility. Journal of the American Academy of Child o Adolescent Psychiatry, 42 (7), 762-769.

Perkins, D. F., \& Hartless, G. (2002). An ecological risk-factor examination of suicide ideation and behavior of adolescents. Journal of Adolescent Research, 17 (1), 3-26.

Plutchik, R. (1995). Outward and inward directed aggressiveness: The interaction between violence and suicidality. Pharmacopsychiatry, 28 (Suppl. 2), 47-57.

Plutchik, R. (2000). Aggression, Violence, and Suicide. In R. Marris, W., A. Berman, L. \& M. Silverman (Eds.), Comprehensive Textbook of Suicidology (407-423). New York: The Guilford Press.

Plutchik, R., Botsis, A. J., \& van Praag, H. M. (1995). Psychopathology, selfesteem, sexual and ego functions as correlates of suicide and violence risk. Archives of Suicide Research, 1 (1), 27-38.

Plutchik, R., \& van Praag, H. M. (1990). Psychosocial correlates of suicide and violence risk. In H. M. van Praag (Ed.), Violence and Suicidality: Perspective in Clinical and Psychobiological Research (37-65). New York: The Guilford Press.

Plutchik, R., van Praag, H. M., \& Conte, H. R. (1989). Correlates of suicide and violence risk: III. A two-stage model of countervailing forces. Psychiatry Research, 28 (2), 215-225.

Portzky, G., Audenaert, K., \& van Heeringen, K. (2005). Suicide among adolescents: A psychological autopsy study of psychiatric, psychosocial and personality-related risk factors. Social Psychiatry and Psychiatric Epidemiology, 40 (11), 922-930.

Prinstein, M. J., Boergers, J., Spirito, A., Little, T. D., \& Grapentine, W. L. (2000). Peer functioning, family dysfunction, and psychological symptoms in a risk factor model for adolescent inpatients' suicidal ideation severity. Journal of Clinical Child Psychology, 29 (3), 392-405.

Pronovost, J., \& Leclerc, D. (2002). L'évaluation et le dépistage des adolescent(e)s suicidaires en centres jeunesse. / Evaluation and tracking of suicidal teenagers in Centres jeunesse. Revue de Psychoéducation et d'Orientation, 31 (1), 81-100. 
Pronovost, J., Leclerc, D., \& Dumont, M. (2003). Facteurs de protection reliés au risque suicidaire chez des adolescents: comparaison de jeunes du milieu scolaire et de jeunes en Centres jeunesse. / Protection factors linked to suicidal risk in adolescents: comparison of school system teenagers and other teens residing in Youth Centers. Revue Québécoise de Psychologie, 24 (1), 179-199.

Putnins, A. L. (2005). Correlates and Predictors of Self-Reported Suicide Attempts Among Incarcerated Youths. International Journal of Offender Therapy and Comparative Criminology, 49 (2), 143-157.

Renaud, J., Berlim, M. T., McGirr, A., Tousignant, M., \& Turecki, G. (2008). Current psychiatric morbidity, aggression/impulsivity, and personality dimensions in child and adolescent suicide: A case-control study. Journal of Affective Disorders, 105 (1-3), 221-228.

Renaud, J., Brent, D. A., Birmaher, B., Chiappetta, L., \& Bridge, J. (1999). Suicide in adolescents with disruptive disorders. Journal of the American Academy of Child $\mathcal{W}$ Adolescent Psychiatry, 38 (7), 846-851.

Renaud, J., Chagnon, F., Turecki, G., \& Marquette, C. (2005). Completed Suicides in a Youth Centres Population. The Canadian Journal of Psychiatry / La Revue canadienne de psychiatrie, 50 (11), 690-694.

Roberts, A. R., \& Bender, K. (2006). Juvenile Offender Suicide: Prevalence, Risk Factors, Assessment, and Crisis Intervention Protocols. International Journal of Emergency Mental Health, 8 (4), 255-266.

Rohde, P., Mace, D. E., \& Seeley, J. R. (1997). The association of psychiatric disorders with suicide attempts in a juvenile delinquent sample. Criminal Behaviour and Mental Health, 7 (3), 187-200.

Rohde, P., Seeley, J. R., \& Mace, D. E. (1997). Correlates of suicidal behavior in a juvenile detention population. Suicide and Life-Threatening Bebavior, 27 (2), 164-175.

Ruchkin, V. V., Schwab-Stone, M., Koposov, R. A., Vermeiren, R., \& King, R. A. (2003). Suicidal ideations and attempts in juvenile delinquents. Journal of Child Psychology and Psychiatry, 44 (7), 1058-1066.

Ryan, J. P., \& Testa, M. F. (2005). Child maltreatment and juvenile delinquency: Investigating the role of placement and placement instability. Children and Youth Services Review, 27 (3), 227-249.

Sanislow, C. A., Grilo, C. M., Fehon, D. C., Axelrod, S. R., \& McGlashan, T. H. (2003). Correlates of suicide risk in juvenile detainees and adolescent inpatients. Journal of the American Academy of Child $\mathcal{N}$ Adolescent Psychiatry, 42 (2), 234-240.

Sourander, A., Helstelä, L., Haavisto, A., \& Bergroth, L. (2001). Suicidal thoughts and attempts among adolescents: A longitudinal 8-year follow-up study. Journal of Affective Disorders, 63 (1-3), 59-66.

Speckens, A. E. M., \& Hawton, K. (2005). Social Problem Solving in Adolescents with Suicidal Behavior: A Systematic Review. Suicide and Life-Threatening Bebavior, 35 (4), 365-387.

St-Laurent, D., \& Gagné, M. (2008). Surveillance de la mortalité par suicide au Québec: Ampleur et évolution du problème de 1981 à 2006. Québec: Institut national de santé publique du Québec. 
Thompson, M. P., Ho, C.-H., \& Kingree, J. B. (2007). Prospective Associations between Delinquency and Suicidal Behaviors in a Nationally Representative Sample. Journal of Adolescent Health, 40 (3), 232-237.

Tychey, C., \& Lighezzolo, J. (2004). L'évaluation de la résilience: quels critères diagnostiques envisager? Perspectives psychiatriques, 43 (3), 226-233.

Vermeiren, R., Schwab-Stone, M., Ruchkin, V. V., King, R. A., Van Heeringen, C., \& Deboutte, D. (2003). Suicidal behavior and violence in male adolescents: A school-based study. Journal of the American Academy of Child $\mathfrak{F}$ Adolescent Psychiatry, 42 (1), 41-48.

Wasserman, G. A., \& McReynolds, L. S. (2006). Suicide Risk at Juvenile Justice Intake. Suicide and Life-Threatening Behavior, 36 (2), 239-249.

Wilson, K. G., Stelzer, J., Bergman, J. N., Kral, M. J., Inayatullah, M., \& Elliott, C.A. (1995). Problem solving, stress, and coping in adolescent suicide attempts. Suicide and Life-Threatening Behavior, 25 (2), 241-252.

Woolf, A., \& Fink, S. G. (1985). Epidemiology of Trauma in a Population of Incarcerated Youth. Pediatrics, 75 (3), 463-468.

Zingraff, M., T., Leiter, J., Myers, K., A., \& Johnsen, M., C. (1993). Child Maltreatment and Youthful Problem Behavior. Criminology, 31 (2), 173-202.

Zlotnick, C., Wolfsdorf, B. A., Johnson, B., \& Spirito, A. (2003). Impaired Self-Regulation and Suicidal Behavior Among Adolescent and Young Adult Psychiatric Inpatients. Archives of Suicide Research, 7 (2), 149-157.

ABSTRACT - Although a marked decrease is observed in recent years, suicide is the second cause of mortality for 15-19 year olds, and the first cause for 20-34 year olds. Juvenile offenders have a higher risk of suicidal behaviours, but risk factors in this population are not well studied. This paper presents the principal risk and protection factors associated with juvenile offender suicide risk by integrating them in an interactive model. Among the factors taken into account in the model are factors related to life events, individual factors (psychopathology, substance abuse, individual and personality characteristics), social factors (family, social network) and living factors (life environment and neighbourhood, school, residential facilities for young offenders). The case study of a teenage young offender - Olivier - is presented in order to support the interactive model.

KEYWORDS - Suicide, adolescent, suicide attempt, suicide ideation, young offender, conceptual model, risk factor.

RESUMEN - Aunque ha disminuido considerablemente en años recientes, el suicidio es la segunda causa de mortalidad para la población de 15 a 19 años y la primera en la de 20 a 34 años. A pesar de que la delincuencia es un factor de riesgo de suicidio adicional entre los jóvenes, los estudios al respecto son todavía escasos. Este artículo presenta los principales factores de riesgo y de protección asociados al suicidio entre los jóvenes infractores, integrando dichos factores en un modelo interactivo. Entre los factores tomados en cuenta en el modelo tenemos los ligados a eventos de sus vidas, los individuales (psicopatología, consumo de sustancias psicotrópicas o alcohol, características individuales y de personalidad), y factores sociales (familia, red social) y los 
factores residenciales (medio de vida y vecindario, escuela, medio residencial para jóvenes infractores). Se presenta también el caso de Olivier, joven infractor, con el fin de ilustrar el funcionamiento del modelo interactivo.

PALABRAS CLAVE - Suicidio, adolescentes, tentativa de suicidio, ideas suicidas, jóvenes infractores, modelo conceptual, factores de riesgo. 